\title{
Purpose in life in patients with rheumatoid arthritis
}

\author{
Pieter J. M. Verduin • Geertruida H. de Bock • \\ Theodora P. M. Vliet Vlieland • Andreas J. Peeters • \\ John Verhoef • Wilma Otten
}

Received: 20 July 2007 /Revised: 11 December 2007 / Accepted: 11 December 2007 /Published online: 24 January 2008

(C) The Author(s) 2007

\begin{abstract}
To evaluate the role of purpose in life among people with rheumatoid arthritis (RA), a questionnaire comprising the Purpose in Life test (PIL) and the purpose in life dimension of the Psychological Well-Being test (PWB-pil) was sent to a random sample of 300 patients with RA. Additional questions comprised sociodemographic and disease characteristics, physical, mental and social functioning, coping (Coping with rheumatic stressors questionnaire), and quality of life (RAND-36). Associations between sociodemographic and disease characteristics, physical, mental and social functioning, and coping on the one side and the two measures of purpose in life on the
\end{abstract}

P. J. M. Verduin

University of Professional Education,

Leiden, The Netherlands

G. H. de Bock

Department of Epidemiology,

University Medical Center Groningen,

Groningen, The Netherlands

T. P. M. Vliet Vlieland $(\bowtie)$

Department of Rheumatology, Leiden University Medical Center, C1-R, P.O. Box 9600, 2300 RC Leiden, The Netherlands e-mail: t.p.m.vliet_vlieland@lumc.nl

\section{A. J. Peeters}

Department of Rheumatology, Reinier de Graaf Gasthuis,

Delft, The Netherlands

J. Verhoef

Department of Physical Therapy,

Leiden University Medical Center,

Leiden, The Netherlands

\section{W. Otten}

Department of Medical Decision Making,

Leiden University Medical Center,

Leiden, The Netherlands other side and associations between the two purpose of life measures and physical and mental dimensions of quality of life were assessed by means of univariate and multivariate regression analyses. The response rate was 156 of 300 (52\%). The median PIL and PWB-pil scores were 103 (range 63-131) and 82 (41-110), respectively. A lower age, a better mental health status, and an optimistic coping style were significantly associated with both higher PIL and PWB-pil scores, whereas more participation in leisure and/ or social activities was associated with a higher PIL score. It was found that the PIL and PWB-pil contributed independently and significantly to the mental component summary scale of the RAND-36. In RA patients, lower age, a better mental health status, an optimistic coping style, and participation in leisure and/or social activities were significantly associated with more sense of purpose in life. Purpose in life pays a significant and independent contribution to the mental component of quality of life. These findings highlight the significance of the concept of purpose in life in patients with RA.

Keywords Adaptation - Arthritis · Health status indicators · Personal satisfaction · Psychological · Quality of life . Rheumatoid · Value of life

\section{Introduction}

Rheumatoid arthritis (RA) is a disease that, despite substantial advances in medical treatment, affects the lives of individuals considerably $[1,2]$. On the individual level, there is wide variability in the level of perceived disability among people with comparable disease severity [3,4]. A number of studies illustrate that the relationships between disease severity and perceived health status are mediated by psychological 
factors, such as coping, anxiety, and depression; however, these relationships vary with respect to their directional nature and appear to be complex and dynamic [5-7].

In the literature on the relationships between physical health status and psychological factors, it is more and more acknowledged that apart from negative psychological states, such as anxiety and depression, positive emotions and subjective states of psychological well being should be taken into account [6, 8, 9]. In this "positive psychology movement," meaning or purpose in life is considered to be an important concept [8-12]. The terms "meaning" and "purpose in life" are often used interchangeably and with varying definitions [12-14]. On the one hand, "meaning" or "purpose in life" is considered to be a trait, an inner strength, as that which is ultimately responsible for the state of one's inner self, as that which has an inner hold on the moral and spiritual self, and as the why or the reason for living that motivates a person's life $[13,15]$. On the other hand, "meaning" or "purpose in life" is also defined as a stable and generalized intention to accomplish something that is at once meaningful to the self and of consequence to the world beyond the self $[12,15]$.

Despite its acknowledged relevance, the concept "to want to reach something in life" was found to be one of the aspects that were found to be missing in the preliminary International Classification of Functioning, Disability and Health (ICF) core set for patients with RA, aiming to describe the typical spectrum of problems in this patient group [16, 17]. Moreover, the literature on a sense of "meaning" or "purpose in life" and its relationship with health status in patients with arthritis is scarce.

In three cross-sectional studies including patients with RA, "meaning" or "purpose in life" was addressed [18-20]. In the studies by Plach et al. [18] and Schleicher et al. [19], purpose in life was considered as an outcome measure. Plach et al. [18] found that purpose in life was predicted by functional status, with social role quality mediating the effect of functional status on purpose in life and moderating the effect of pain on purpose in life, whereas Schleicher et al. [19] found significant univariate associations between pain and fatigue on the one side and purpose in life on the other side. Mangelli et al. [20] reported significant bivariate correlations between both anxiety and depression and purpose in life, with no contribution of purpose in life to affective disturbance as the main outcome. In none of these studies, the role of coping was taken into account, despite the fact that passive coping has been associated with higher levels of pain, physical disability, and depression in patients with RA $[5,7]$. In all three studies [18-20], the same instrument to measure purpose in life was used [11, 12].

The variety regarding the analyses in these four studies, with purpose in life being considered as an outcome measure, a predictor of physical health status, or a mediator in the relation between disease severity and physical health status, may reflect the absence of a well-grounded theoretical framework, incorporating the role of purpose in life in patients with arthritis. As the literature on purpose in life is scarce and the results of the available studies in part are conflicting, the aim of this exploratory, cross-sectional study was first to determine the relationships between various sociodemographic variables, disease characteristics, and measures of physical, mental, and social functioning, coping with pain, limitations and dependence, and purpose in life in patients with RA, with purpose in life being considered as the outcome and measured by means of two different instruments. Moreover, this study addresses the question to what extent sense of purpose in life adds to the concept of physical and mental dimensions of quality of life, while taking into account all other variables. The results of these analyses may form the basis of further research in the area of purpose in life in this patient group.

\section{Materials and methods}

Study design and patient recruitment

For participation in this cross-sectional study, 300 subjects with RA were randomly selected from the patient registries of the outpatient rheumatology departments of the Leiden University Medical Center, Leiden, and the Reinier de Graaf Gasthuis, Delft, The Netherlands. After verification of the diagnosis of RA according to the criteria of the American College of Rheumatology [21], patients were sent a letter signed by their treating rheumatologist, inviting them to participate in the study, an information leaflet, a questionnaire, and a prestamped envelope. Information on disease duration was derived from the medical record. The medical ethics committees of the two participating hospitals approved the study protocol, and all patients provided written informed consent.

\section{Sociodemographic characteristics}

Sociodemographic data included age, sex, status of living (living with a partner yes/no), education level, working status (paid job yes/no) and active religious affiliation (yes/no).

General disease characteristics

Disease characteristics included disease duration and the patient's global assessments of disease activity, pain, and fatigue as measured with three horizontal visual analog scales (VAS, $0-10 \mathrm{~cm}$ ). The anchors on the left were no disease activity, no pain, and no fatigue, whereas the anchors 
on the right were severe disease activity, pain, and severe fatigue. Thus, higher scores indicate a worse health status.

Physical, mental, and social functioning and quality of life

To assess physical functioning, a Dutch version of the "Health Assessment Questionnaire" (HAQ) was included [22]. The HAQ is a 20 -item instrument assessing a respondent's ability to complete everyday tasks in eight areas including dressing and grooming, rising, eating, walking, personal hygiene, reach, grip, and other activities. Each item is assigned a score ranging from 0 "without difficulty" to 3 "unable to do", which is further adjusted to account for the use of any aids, devices, or help from another person. These are then summed per dimension and averaged to give an overall score between 0.0 (best possible function) to 3.0 (worst function).

Various aspects of physical, mental, and social functioning were measured using the Dutch version of the RAND 36-item Health Survey [23]. The RAND-36 is a generic measure of quality of life, including the same items as the Medical Outcomes Study Short-Form, and although the scoring procedures are somewhat different, the effects on final scores are minimal [24]. The RAND-36 comprises the following subscales: physical functioning, social functioning, role limitations physical functioning, role limitations emotional problems, mental health, vitality, pain, and general health perception. Each subscale ranges from 0 to 100 with higher scores indicating better health status. For some analyses in this study, the subscales of the RAND-36 were categorized over the following constructs: disease characteristics, physical functioning, mental functioning, and role limitations. From the RAND-36, two summary scales were computed: the mental component summary scale and the physical component summary scale. Both summary scales range from 0 to 100 , with higher scales indicating better quality of life.

\section{Coping}

Coping strategies were assessed by means of the "Coping with rheumatic stressors" questionnaire (CORS), an arthritisspecific coping questionnaire, which measures eight coping strategies directed at the most important chronic stressors of inflammatory rheumatic disease: pain, limitations, and dependency, which was validated in patients with RA [25]. Our study included items of the CORS representing the following coping styles related to pain: comforting cognitions (nine items), decreasing activity (eight items), and diverting attention (eight items). Coping styles related to limitations included: optimism (five items), pacing (ten items), and creative solution seeking (eight items). Coping styles with respect to dependence included accepting (six items) and consideration (seven items). For each item, patients reported how often they made use of that particular coping strategy $(1=$ seldom or never, $2=$ sometimes, $3=$ often, $4=$ very often $)$.

Purpose in life

In the literature, several instruments to assess global perceptions of purpose or meaning in life can be found $[26,27]$. We selected the Purpose In Life Test (PIL Test) $[28,29]$ and the purpose in life subscale of the Psychological Wellbeing Scale (PWB-Pil) [11] because of their theoretical underpinnings and their relatively frequent usage in the literature. The PIL originates from the work of Frankl [13] and is intended as a measure of Frankl's basic concept, "the will to meaning," whereas the PWB-pil stems from the psychological wellbeing tradition $[11,12,30]$. Both questionnaires comprise statements about the experience of meaning of life and the attainment of life goals (irrespective of health status or other characteristics) rather than the actual engagement in meaning giving or purposeful activities. However, the PIL items mainly pertain to whether or not a person has life goals (decision or indecision in purpose in life), whereas the PWB-pil mainly comprises items about a person's experience with respect to the presence or absence of life goals.

The PIL Test With this 20-item instrument, respondents are required to denote the extent to which their views of life correspond to points on a seven-point Likert scale for each item. Examples items are: "In life I have: no goals or aims at all (1)-very clear goals and aims (7)" or "I have discovered no mission or purpose in life (1)-clear-cut goals and a satisfying life purpose (7)." The test was translated into Dutch by our own group according to guidelines for translation and cross-cultural adaptation of self-report measures [31]. The total score ranges from 20 to 140, with higher scores indicating a stronger sense of purpose in life. It is assumed that PIL raw scores from 92 through 112 are in the indecisive range; scores above 112 indicate the presence of definite purpose and meaning in life; scores below 92 indicate the lack of clear meaning and purpose $[28,29]$.

The purpose in life subscale of the Psychological Wellbeing Scale The PWB is a self-rating inventory that covers six areas of wellbeing: autonomy, environmental mastery, personal growth, positive relations with others, purpose in life, and self-acceptance [30]. We used the full 20-item version of the purpose in life subscale of a Dutch translation of the PWB (Arrindell, personal communication), using the original six-point Likert scale format. Every item score ranges from " 1 " to " 6 ," with the total score ranging from 20 to 120 and higher scores indicating a stronger sense of purpose in life. Example items from the PWB-pil are: "I 
enjoy making plans for the future and working to make them reality" (1-6) or "My daily activities often seem trivial and unimportant to me" (1-6) [11, 12, 30].

\section{Statistical analysis}

All analyses were conducted using SPSS 12.0 for Windows (SPSS, Chicago, IL). In case of missing data for the PIL or PWB-pil, the mean score of the other items for that person was used to calculate a total score for that person within that dimension with the maximum number of missing items set at $25 \%$. In case of more missing items, the total score could not be computed. The internal consistency of the two instruments was computed by means of Cronbach's alpha and their mutual correlation by Spearman rank correlation coefficient.

With respect to the basic sociodemographic and disease characteristics, categorical data were described as numbers and percentages and continuous data as median and range. Differences regarding the basic sociodemographic and disease characteristics between the two centers involved were analyzed using the Mann-Whitney $U$ or Chi-squared test where appropriate.

To determine which variables were associated with purpose in life, univariate multiple linear regression analyses were performed, with the PIL test and the PWB-pil being the dependent variables and the results expressed as $B$ with the $95 \%$ confidence interval. The following constructs were used as independent variables: sociodemographic characteristics (age, sex, status of living, education, work status, and active religious affiliation), disease characteristics (disease duration, VAS pain, disease activity and fatigue, and the RAND-36 pain, vitality and general health perception subscales), physical functioning (HAQ, RAND-36 physical functioning subscale), mental health (RAND-36 mental functioning subscale), role limitations (RAND-36 social functioning, role limitations physical functioning and role limitations emotional problems subscales, and participation in social and/or leisure activities), and coping.

Because the aim of this study was to study coping strategies in general instead of studying coping strategies directed at specific chronic stressors of inflammatory rheumatic disease (pain, limitations, and dependency), a factor analysis was performed on all items of the CORS. The aim of this factor analysis was to reduce the CORS items to a limited number of meaningful dimensions for this study. The internal consistency of the resulting dimensions was determined by computing Cronbach's alpha.

Variables or constructs that were statistically significantly associated with measures of purpose in life in the univariate analyses were then entered into multivariate multiple linear regression models with the same dependent variables (method stepwise backward).
To determine whether the concept of purpose in life independently contributes to quality of life, univariate analyses were performed, with the RAND-36 Physical and Mental Component Summary Scales as dependent variables. Conditional on either the PIL or the PWB-pil, all other variables or constructs were entered into univariate regression analyses. All independent variables that were significantly associated with measures of physical and mental health status were then, again conditional on either the PIL or the PWB-Pil, entered into multivariate models with the RAND-36 Physical and Mental Component Summary Scales as dependent variables.

A $p$ value less than 0.05 was considered as statistically significant in all analyses. All analyses were repeated with a $p$ value of less than 0.10 as a threshold for entering the multivariate multiple regression models.

\section{Results}

Of the 300 questionnaires, 116 were returned within 3 weeks. One-hundred and thirty-seven of the 184 initial nonresponders could be contacted by telephone by the principal investigator (Verduin). Fifty-three of them did send in the questionnaire at a later stage. In total, 13 of the 169 returned questionnaires were left blank, yielding a total response rate of 156 of 300 (52\%). The response rates were 84 of $150(56 \%)$ in the Leiden University Medical Center and 72 of $150(48 \%)$ in the Reinier de Graaf Gasthuis, respectively. There were no statistically significant differences between the sociodemographic and disease characteristics among the two centers (data not presented).

The characteristics of the patients and their disease are shown in Table 1. One-hundred and forty-seven subjects (94\%) were born in The Netherlands, and 152 subjects (97\%) had the Dutch nationality. In all, the median age, the sex distribution, the median disease duration, and median HAQ score indicate that our sample represents a typical outpatient population of RA patients.

In seven patients $(4.5 \%)$, the PIL and PWB-pil scores could not be computed because six or more items were missing. The mean and median scores of the remaining 149 patients were 102.4 (SD 13.0) and 103 (range 63-131) for the PIL and 81.6 (SD 15.5) and 82 (range 41-110) for the PWB-pil. For none of the characteristics, a significant difference between the two hospitals was observed (results not shown).

Cronbach's alphas for the PIL and PWB-pil were 0.83 and 0.81 , respectively. The correlation between the PIL and the PWB-pil was good (Spearman's $r=0.62, p<0.001$ ).

Table 2 shows the univariate associations between various on the one side and the two measures of purpose in life on the other side. 
Table 1 Characteristics of 156 patients with rheumatoid arthritis participating in a study on purpose in life
Results are expressed as median (range), unless specified otherwise.

${ }^{a}$ Education level was divided into three categories based on the Dutch school system: primary education, $0-8$ years; secondary education, 9-16 years; and higher vocational education/university, 17 years and more.
Characteristics

\begin{tabular}{|c|c|}
\hline \multicolumn{2}{|l|}{ Sociodemographic characteristics } \\
\hline Age, years $(n=156)$ & $60(24-87)$ \\
\hline Male patients, $n(\%)(n=156)$ & $48(31)$ \\
\hline Living with partner, $n(\%)(n=152)$ & $127(84)$ \\
\hline High educational level ${ }^{\mathrm{a}}, n(\%)(n=155)$ & $52(34)$ \\
\hline Paid job, $n(\%)(n=152)$ & $46(30)$ \\
\hline Active religious affiliation, $n(\%)(n=152)$ & $55(36)$ \\
\hline \multicolumn{2}{|l|}{ Disease characteristics } \\
\hline Duration of disease, years $(n=152)$ & $10(0-49)$ \\
\hline \multicolumn{2}{|l|}{ Visual analog scales patient, $0-10 \mathrm{~cm}$} \\
\hline Disease activity $(n=155)$ & $3.5(0-10)$ \\
\hline Pain $(n=152)$ & $3.5(0-10)$ \\
\hline Fatigue $(n=154)$ & $4.8(0-10)$ \\
\hline RAND-36 vitality $(n=150)$ & $60.0(5.0-100)$ \\
\hline RAND-36 pain $(n=147)$ & $67.3(0-100)$ \\
\hline RAND-36 general health perception $(n=150)$ & $52.5(10.0-100)$ \\
\hline \multicolumn{2}{|l|}{ Physical functioning } \\
\hline Health Assessment Questionnaire $(n=156)$ & $0.75(0-3.0)$ \\
\hline RAND-36 Physical functioning $(n=156)$ & $71.3(0-100)$ \\
\hline \multicolumn{2}{|l|}{ Mental functioning } \\
\hline RAND-36 Mental Health $(n=149)$ & $76.0(16.0-100)$ \\
\hline \multicolumn{2}{|l|}{ Role limitations } \\
\hline RAND-36 social functioning ( $n=146)$ & $75.0(0-100)$ \\
\hline RAND-36 role limitations physical functioning $(n=155)$ & $75.0(0-100)$ \\
\hline RAND-36 role limitations emotional problems $(n=153)$ & $100(0-100)$ \\
\hline Participation in leisure and/or social activities, $n(\%)(n=155)$ & $132(85)$ \\
\hline \multicolumn{2}{|l|}{ Coping with rheumatic stressors } \\
\hline Pain - comforting cognitions $(n=143)$ & $28.0(10.0-36.0)$ \\
\hline Pain—Decreasing activity $(n=139)$ & $19.0(8.0-31.0)$ \\
\hline Pain-Diverting attention $(n=139)$ & $18.0(8.0-32.0)$ \\
\hline Limitations-optimism $(n=150)$ & $16.0(5.0-20.0)$ \\
\hline Limitations-pacing $(n=148)$ & $27.0(10.0-39.0)$ \\
\hline Limitations - creative solutions $(n=143)$ & $21.0(8.0-32.0)$ \\
\hline Dependence-accepting $(n=148)$ & $14.0(6.0-24.0)$ \\
\hline Dependence - consideration $(n=144)$ & $20.0(7.0-28.0)$ \\
\hline \multicolumn{2}{|l|}{ Purpose in life } \\
\hline Purpose in Life test $(n=149)$ & $103(63-131)$ \\
\hline PWB-pil $(n=149)$ & $82(41-110)$ \\
\hline
\end{tabular}

Regarding the factor analysis on the CORS, we subtracted four dimensions with satisfying reliabilities: seeking solutions (30 items, Cronbach's alpha=0.92), positivity (16 items; Cronbach's alpha $=0.83$ ), resilience (eight items, Cronbach's alpha $=0.83$ ), and distraction (seven items, Cronbach's alpha=0.73).

It appeared that sex, living status, disease duration, the VAS disease activity, and the coping dimensions seeking solutions, resilience, and distraction were not statistically significantly associated with any of the two measures of purpose in life.

Table 3 shows the results of the multivariate regression analyses with the PIL and PWB-pil scores as dependent variables. It was found that a younger age, a better mental health, and an optimistic coping style were significantly associated with both measures of purpose in life. Moreover, participation in leisure/social activities was associated with a higher PIL score.

Table 4 shows the results of the multivariate analyses, performed to examine whether purpose in life measures add to the prediction of physical and mental dimensions of quality of life, above and beyond all other sociodemographic and disease characteristics and coping. Apart from either the PIL or the PWB-pil, all variables that were found to be significantly associated with the RAND-36 Mental or Physical Summary Scales in the univariate analyses (conditional on PIL or PWB-pil) were entered in the multivariate models. The PIL and the PWB-pil were, 
Table 2 Univariate associations of sociodemographic and disease characteristics, measures of physical, mental, and social functioning, and coping with two measures of purpose in life in 149 patients $^{\mathrm{a}}$ with rheumatoid arthritis (values expressed as $B$ with the $95 \%$ confidence interval)
${ }^{*} p<0.05,{ }^{*} p<0.005$, univariate regression analysis

${ }^{\text {a }}$ In seven patients, the PIL and PWB-pil scores could not be computed because of missing data.

${ }^{\mathrm{b}}$ Dimensions substracted by means of factor analysis on the CORS (Coping with Rheumatic Stressors).
Purpose in Life Test (PIL), Psychological Wellbeing-Purpose in $B(95 \% \mathrm{CI})$

\begin{tabular}{|c|c|c|}
\hline \multicolumn{3}{|l|}{ Sociodemographic characteristics } \\
\hline Age & $-0.23(-0.39--0.06)^{*}$ & $-0.40(-0.59--0.21)^{* *}$ \\
\hline Male patient & $0.00(-4.65-4.64)$ & $-0.37(-6.00-5.25)$ \\
\hline Living with partner & $0.47(-5.79-6.73)$ & $0.39(-7.18-7.96)$ \\
\hline High educational level & $3.28(-1.21-7.76)$ & $11.42(6.29-16.54)^{* *}$ \\
\hline Paid job & $5.70(1.13-10.27)^{*}$ & $9.58(4.19-14.96)^{* *}$ \\
\hline Active religious affiliation & $3.16(-1.37-7.70)$ & $6.26(0.87-11.66)^{*}$ \\
\hline \multicolumn{3}{|l|}{ Disease characteristics } \\
\hline Duration of disease & $-0.28(-0.69-0.12)$ & $-0.21(-0.72-0.31)$ \\
\hline \multicolumn{3}{|l|}{$\begin{array}{l}\text { Visual analog scales patient, } \\
0-10 \mathrm{~cm}\end{array}$} \\
\hline Disease activity & $-0.61(-1.36-0.14)$ & $0.55(-1.46-0.37)$ \\
\hline Pain & $-1.15(-1.96--0.34)^{* *}$ & $1.05(-2.04--0.05)^{*}$ \\
\hline Fatigue & $-0.98(-1.65--0.30) * *$ & $-0.98(-1.81--0.15)^{*}$ \\
\hline RAND-36 vitality & $0.22(0.11-0.32)^{* *}$ & $0.27(0.14-0.39)^{* *}$ \\
\hline RAND-36 pain & $0.15(0.05-0.24)^{* *}$ & $0.16(0.04-0.27)^{*}$ \\
\hline $\begin{array}{l}\text { RAND-36 general health } \\
\text { perception }\end{array}$ & $0.16(0.07-0.26)^{* *}$ & $0.16(0.04-0.29)^{*}$ \\
\hline \multicolumn{3}{|l|}{ Physical functioning } \\
\hline RAND-36 physical functioning & $0.13(0.05-0.20)^{* *}$ & $0.17(0.08-0.27)^{* *}$ \\
\hline Health assessment questionnaire & $-5.59(-8.82-2.35)^{* *}$ & $-7.00(-10.92--3.10) * *$ \\
\hline \multicolumn{3}{|l|}{ Mental functioning } \\
\hline RAND-36 mental health & $0.34(0.24-0.45)^{* *}$ & $0.33(0.20-0.47)^{* *}$ \\
\hline \multicolumn{3}{|l|}{$\begin{array}{l}\text { Role limitations societal } \\
\text { participation }\end{array}$} \\
\hline RAND-36 social functioning & $0.16(0.07-0.25)^{* *}$ & $0.13(0.02-0.25)^{*}$ \\
\hline $\begin{array}{l}\text { RAND-36 Role limitations } \\
\text { physical functioning }\end{array}$ & $0.07(0.02-0.12)^{*}$ & $0.06(-0.01-0.12)$ \\
\hline $\begin{array}{l}\text { RAND-36 Role limitations } \\
\text { emotional problems }\end{array}$ & $0.09(0.04-0.15)^{* *}$ & $0.09(0.02-0.15)$ \\
\hline $\begin{array}{l}\text { Participation in leisure/social } \\
\text { activities }\end{array}$ & $10.53(4.63-16.43) * *$ & $9.32(2.00-16.64) *$ \\
\hline \multicolumn{3}{|l|}{ Coping $^{\mathrm{b}}$} \\
\hline Seeking solutions & $2.72(-1.68-7.13)$ & $0.26(-5.12-5.64)$ \\
\hline Positivity & $13.92(9.14-18.71)^{* *}$ & $14.11(8.11-20.11)^{* *}$ \\
\hline Resilience & $1.28(-2.27-4.83)$ & $-2.81(-7.10-1.48)$ \\
\hline Distraction & $0.54(-3.56-4.63)$ & $-0.96(-5.91-3.99)$ \\
\hline
\end{tabular}

apart from other variables, found to contribute significantly to the RAND-36 Mental Health Summary Scale but not to the RAND-36 Physical Health Summary Scale.

\section{Discussion}

In this cross-sectional study in patients with RA, it was found that lower age, better mental health status, an optimistic coping style, and participation in social and/or leisure activities were significantly associated with more sense of purpose in life. Moreover, it was found that purpose in life paid an independent contribution to mental health status.

In our study, median scores of 102.4 and 81.6 were obtained for the PIL and PWB-pil, respectively. For the
PIL, no data regarding other populations with RA patients are available for comparison. With respect to the average PWB-pil score, the results of the present study cannot be directly compared with the PWB-pil scores observed in RA patients in previous studies [18-20], as in those studies, 14-item versions of the PWB-pil subscale were used. If the mean scores are however expressed as a percentage of the maximum score, the percentage score of 81.6 of $120=68 \%$ as found in the present study is similar to the $69 \%$ observed by Mangelli et al. [20] and lower than the 74 and $82 \%$ as described by Plach et al. [18] and Schleicher et al. [19], respectively. In comparison with the results of the PWB-pil scores found in patients with osteoarthritis [32] and healthy populations [30], the findings in RA patients appear to be in the lower ranges, indicating that RA patients feel less 
Table 3 Multivariate associations of sociodemographic and disease characteristics, measures of physical, mental, and social functioning, and coping with two measures of purpose in life in 149 patients $^{\mathrm{a}}$ with rheumatoid arthritis

Purpose in Life Test (PIL),

$B(95 \% \mathrm{CI})$
Psychological Wellbeing-Purpose in life (PWB-pil), B (95\% CI)

Sociodemographic characteristics

Age

$-0.17(-0.33--0.01)^{*}$

$0.23(0.12-0.35)^{* *}$

$6.92(0.69-13.15)^{*}$

Leisure/social activities

Coping ${ }^{\mathrm{b}}$

Positivity
$-0.36(-0.56--0.15)^{* *}$

$0.30(0.14-0.45)^{* *}$

$8.86(2.37-15.35)^{* *}$

${ }^{*} p<0.05, * * p<0.005$, multivariate regression analysis

${ }^{\text {a }}$ In seven patients, the PIL and PWB-pil scores could not be computed because of missing data.

${ }^{\mathrm{b}}$ Dimension subtracted by means of factor analysis on the CORS (Coping with rheumatic stressors).

purpose in life than these groups. The validity of direct comparisons among populations must, however, be done with caution, as the number of subjects as well as their selection and the number of items and the calculation of the scores may vary widely.

To date, little is known about the relationship between sociodemographic and disease characteristics, physical, mental and social functioning, coping with pain, limitations and dependence, and sense of purpose in life in patients with RA. With respect to the univariate associations between sociodemographic characteristics and purpose in life, the results of the present and previous studies vary. The previously described positive association between a higher education level on the one side and more purpose in life on the other side [18] was confirmed in the present study. However, in the present study, a relationship between lower age and more purpose in life was seen, whereas both in the studies by Mangelli et al. [20] and Plach et al. [18], no association between age and purpose in life was observed.

With respect to social functioning, Plach et al. [18] found an association between a better social role quality and more purpose in life, which is likely to be in accordance with the positive relationship between paid employment and social and leisure activities on the one side and purpose in life on the other side as found in the present study. In addition, the univariate positive correlation between the RAND-36 social functioning dimension and purpose in life as observed in the present study points into the same direction.
Table 4 Multivariate associations of sociodemographic, disease characteristics, physical functioning, and coping, conditional on either the PIL or PWB-pil ${ }^{\text {a }}$, with RAND summary scales physical health and mental health as dependent variables in 149 patients $^{\mathrm{a}}$ with rheumatoid arthritis

Only significant results are presented and expressed as $B$ with the $95 \%$ confidence interval. ${ }^{*} p<0.05, * * p<0.005$, multivariate regression analysis ${ }^{\mathrm{a}}$ In seven patients, the PIL and PWB-pil scores could not be computed because of missing data.

${ }^{\mathrm{b}}$ Dimensions substracted by means of factor analysis on the CORS (Coping with rheumatic stressors).

\begin{tabular}{|c|c|c|}
\hline & $\begin{array}{l}\text { RAND summary scale } \\
\text { physical health }\end{array}$ & $\begin{array}{l}\text { RAND summary scale } \\
\text { mental health }\end{array}$ \\
\hline PIL & $0.12(-0.08-0.31)$ & $0.43(0.20-0.64)^{* *}$ \\
\hline \multicolumn{3}{|l|}{ Disease characteristics and coping } \\
\hline \multicolumn{3}{|l|}{ Visual analogue scales patient } \\
\hline Fatigue & $-2.38(-3.24--1.53)^{* *}$ & $-1.96(-2.95--0.97) * *$ \\
\hline \multicolumn{3}{|l|}{ Coping ${ }^{\mathrm{b}}$} \\
\hline CORS Positivity & $7.86(1.65-14.06)^{*}$ & \\
\hline CORS Resilience & $-6.99(-11.41--2.57) * *$ & $-6.88(-11.85--1.91)^{* *}$ \\
\hline CORS Seeking solutions & $-8.88(-14.55--3.20) * *$ & \\
\hline PWB-pil & $0.02(-0.14-0.18)$ & $0.28(0.08-0.47)^{*}$ \\
\hline \multicolumn{3}{|l|}{ Disease characteristics and coping } \\
\hline \multicolumn{3}{|l|}{ Visual analogue scales patient } \\
\hline Pain & $-1.12(-2.18--0.07)^{*}$ & \\
\hline Fatigue & $-2.20(-3.11--1.29) * *$ & $-2.08(-3.15--1.00) * *$ \\
\hline \multicolumn{3}{|l|}{ Coping $^{\mathrm{b}}$} \\
\hline CORS Positivity & $8.02(1.84--14.19)^{*}$ & \\
\hline CORS Resilience & $-6.24(-10.66--1.83) * *$ & $-6.45(-11.84--1.06)^{*}$ \\
\hline CORS Seeking solutions & $-7.90(-13.54--2.26)^{* *}$ & \\
\hline
\end{tabular}


In our study, a positive association between active religious affiliation and purpose in life was found for the PWB-pil but not the PIL. The relationship between religion and purpose and life was not addressed in earlier studies on purpose in life in RA [18-20]. In a previous study on religious and nonreligious coping methods among persons with RA, it was found that apart from nonreligious coping, religious coping makes a unique contribution to coping with RA [33]. Moreover, religious coping appears to have an emotional rather than a problem-solving focus [33]. In another study, it was found that spirituality, which may in part overlap with religious practice, may facilitate emotional adjustment and resilience in people with RA, by experiencing more positive feelings and attending to positive elements of their lives [34]. In our study, a clear relationship between an optimistic coping style and purpose in life was seen. Coping was not taken into account in previous studies on the role of purpose in life in RA [18-20].

The relationship between better physical functioning and less pain and fatigue with more purpose in life as observed in the present study was also seen in the studies by Plach et al. [18] and Schleicher et al. [19] but was absent in the study by Mangelli et al. [20].

Our multivariate analyses with purpose in life considered as the outcome are also difficult to compare with those of previous studies in RA [18-20], as in these studies, different statistical models were used.

Our study also focused specifically on the relationship between purpose in life and quality of life, showing that purpose in life uniquely adds to the mental dimension of quality of life.

However, the cross-sectional design and the statistical methods employed in this study do not allow for drawing conclusions about the direction of the relationships or developing complex, dynamic models. Therefore, it remains unclear whether sense of purpose in life should be considered as an outcome or as a determinant of various aspects of health status or quality of life. The question remains whether an optimistic coping style, better mental functioning, and social engagements result in more sense of purpose in life or that purpose in life should rather be considered as a personality trait, with more sense of purpose in life leading to better mental and social functioning and employing more constructive coping strategies. In addition, apart from the question whether variables should be considered to be determinants or outcomes, potential mediating roles of any of the variables we studied could not be examined.

Another limitation of our study is in the instruments used to measure purpose in life. In this study, the two instruments had similar internal consistency and correlated moderately well. However, the items of the PIL and PWB-pil had a slightly different focus, with the PIL mainly containing statements on the presence or absence of life goals and the PWB-pil also comprising items on the person's experience regarding the sense of meaning or purpose. This observation may be connected to the different origins of the questionnaires, with the PIL originating from the work of Frankl [13] and the PWB-pil from the psychological wellbeing tradition [11, 12, 30]. According to Ryan and Deci [35], the conceptualizations of wellbeing stem from two traditions in the psychological literature, focusing on eudaimonic wellbeing or hedonic wellbeing. Ryff's work on the PWB $[11,12,30]$ exemplifies the eudaimonic tradition, with wellbeing being described as the extent to which people endorse high levels of autonomy, environmental mastery, personal growth, positive relations with others, purpose in life, and self-acceptance, whether accompanied by feeling good or not. Given the different origins of the questionnaires, it is conceivable that the PIL and PWB-pil do not exactly represent the same aspects of purpose in life.

As both instruments clearly focused on life goals and purpose in life and did not comprise items about the actual engagement in potentially meaningful activities (e.g., paid employment, charity), it is conceivable that their contents are clearly distinct from the concept of social functioning. As we have no gold standard for purpose in life, it is difficult to determine which of the two instruments is the most valid measure to reflect purpose in life.

Despite its limitations, we think that this study indicates that the concept of purpose in life in RA patients warrants further research. In the qualitative validation study on the ICF RA core set [17], it was noted by patients that the concept of purpose in life was not explicitly addressed, where it was by patients considered as a relevant topic. The concept of purpose in life could probably be assigned to the not-yet-developed ICF component personal factors.

Concerning the practical application of purpose in life measurements in future research, both the instruments we used comprised 20 items and may take some time to complete, which can be an obstacle to use these instruments in research and clinical practice. Recently, even shorter instruments than the previously mentioned 14 -item versions of the PWB-pil subscale [18-20] have been developed, such as the four-item "meaning in life" subscale of the "spiritual, religion, and personal beliefs" scale [36], the sixitem Life Engagement test [37], and a six-item version of PWB-pil [38]. None of these instruments have so far been used in arthritis care research. A consensus regarding which instrument to use to assess purpose in life in this field would advance this line of research.

In this study, a response rate of $52 \%$ was obtained. As of the nonresponders, no sociodemographic or clinical data were gathered; it remains unclear to what extent the relatively high level of nonresponse might affect the external validity of our results. 
How can these results be used by healthcare providers? Inquiry about the sense of purpose in life may require time and skills of healthcare providers. However, the observation from this study that there are, apart from personal characteristics such as age and educational level, a number of potentially modifiable factors associated with the sense of a purpose in life suggests that it is worthwhile to take into account. Problems with the sense of purpose in life may reflect other emotional problems, so that referral to a psychological intervention is worth considering, as psychological interventions including cognitive behavioral therapy have been found to improve RA patients' coping [39] and psychological status [39, 40]. Our findings suggest that purpose in life merits detailed investigation in future studies, especially those focusing on mental and social functioning, quality of life, and psychological interventions in RA patients.

Acknowledgments The authors are grateful to R. Pasterkamp, I.G. de Boer and M.H. van den Berg for supporting the conduct of this study.

Open Access This article is distributed under the terms of the Creative Commons Attribution Noncommercial License which permits any noncommercial use, distribution, and reproduction in any medium, provided the original author(s) and source are credited.

\section{References}

1. Scott DL, Smith C, Kingsley G (2005) What are the consequences of early rheumatoid arthritis for the individual? Best Pract Res Clin Rheumatol. 19(1):117-136 (Feb)

2. Symmons D, Tricker K, Harrison M, Roberts C, Davis M, Dawes P, Hassell A, Knight S, Mulherin D, Scott DL (2006) British rheumatoid outcome study group. Patients with stable long-standing rheumatoid arthritis continue to deteriorate despite intensified treatment with traditional disease modifying anti-rheumatic drugs - results of the British Rheumatoid Outcome Study Group randomized controlled clinical trial. Rheumatology (Oxford) 45 (5):558-565 (May)

3. Hewlett S, Smith AP, Kirwan JR (2002) Measuring the meaning of disability in rheumatoid arthritis: the Personal Impact Health Assessment Questionnaire (PI HAQ). Ann Rheum Dis. 61(11): 986-993 (Nov)

4. Lempp H, Scott D, Kingsley G (2006) The personal impact of rheumatoid arthritis on patients' identity: a qualitative study. Chronic Illn 2:109-120

5. Covic T, Adamson B, Hough M (2000) The impact of passive coping on rheumatoid arthritis pain. Rheumatology 39:1027-1030

6. Walker JG, Jackson HJ, Littlejohn GO (2004) Models of adjustment to chronic illness: using the example of rheumatoid arthritis. Clin Psychol Rev 24:461-488

7. Treharne GJ, Lyons AC, Booth DA, Kitas GD (2007) Psychological well-being across 1 year with rheumatoid arthritis: coping resources as buffers of perceived stress. Br J Health Psychol 12:323-345

8. Myers DG, Diener E (1996) The pursuit of happiness. Sci Am 274 (5):70-72 (May)

9. Lucas RE, Diener E, Suh E (1996) Discriminant validity of wellbeing measures. J Pers Soc Psychol 71:616-628
10. Diener E (2000) Subjective well-being. The science of happiness and a proposal for a national index. Am Psychol 55:34-43

11. Ryff CD (1989) Happiness is everything, or is it? Exploration on the meaning of psychological well-being. J Pers Soc Psychol 57:1069-1083

12. Ryff CD, Singer B (1998) The contours of positive human health. Psychol Inq 9:1-28

13. Frankl VE (1972) The feeling of meaninglessness: a challenge to psychotherapy. Am J Psychoanal 32(1):85-89

14. Wong PTP, Fry PS (eds) (1998) In: The human quest for meaning: a handbook of psychological research and clinical applications. Erlbaum, Mahwah, NJ

15. Damon W, Menon J, Cotton Bronk K (2003) The development of purpose during adolescence. Appl Dev Sci 7:119-128

16. Stucki G, Cieza A, Geyh S, Battistella L, Lloyd J, Symmons D et al (2004) ICF Core Sets for rheumatoid arthritis. J Rehabil Med 44 (Suppl):87-93

17. Stamm TA, Cieza A, Coenen M, Machold KP, Nell VPK, Smolen JS, Stucki G (2005) Validating the International Classification of Functioning, Disability and Health Comprehensive Core Set for Rheumatoid Arthritis form the patient perspective: a qualitative study. Arthritis Rheum 53:431-439

18. Plach SK, Heidrich SM, Waite RM (2003) Relationship of social role quality to psychological well-being in women with rheumatoid arthritis. Res Nurs Health 26:190-202

19. Schleicher H, Alonso C, Shirtcloff EA, Muller D, Loevinger BL, Coe CL (2005) In the face of pain: The relationship between psychological well-being and disability in women with fibromyalgia. Psychother Psychosom 74:231-239

20. Mangelli M, Gribbin N, Büchi S, Allard S, Sensky T (2002) Psychological well-being in Rheumatoid Arthritis: Relationship to 'disease' variables and affective disturbance. Psychother Psychosom $71: 112-116$

21. Arnett FC, Edworthy SM, Bloch DA, McShane DJ, Fries JF, Cooper NS, Healey LA, Kaplan SR, Liang MH, Luthra HS et al (1988) The American rheumatism association 1987 revised criteria for the classification of rheumatoid arthritis. Arthritis Rheum 31:315-324

22. Siegert CE, Vleming LJ, Vandenbroucke JP, Cats A (1984) Measurement of disability in Dutch rheumatoid arthritis patients. Clin Rheumatol 3:305-309

23. Van der Zee KI, Sanderman R, Heyink J (1996) A comparison of two multidimensional measures of health status: The Nottingham Health Profile and the RAND 36-item Survey 1.0. Qual Life Res $5: 165-174$

24. Ware JE Jr, Gandek B (1998) Overview of the SF-36 health survey and the International Quality of Life Assessment (IQOLA) project. J Clin Epidemiol 51:903-912

25. Van Lankveld W, van 't Pad Bosch P, van de Putte L, Naring G, van der Staak C (1994) Disease-specific stressors in rheumatoid arthritis: coping and well-being. Br J Rheumatol 33:1067-1073

26. Reker GT, Fry PS (2003) Factor structure and invariance of personal meaning measures in cohorts of younger and older adults. Pers Individ Diff 35:977-993

27. White CA (2004) Meaning and its measurement in psychosocial oncology. Psychooncology 13:468-481

28. Crumbaugh JC, Maholick LT (1964) An experimental study in existentialism: the psychometric approach to Frankl's concept of noogenic neurosis. J Clin Psychol 20:200-207

29. Crumbaugh JC, Maholick LT (1963) The case for Frankl's 'Will to meaning'. J Existent Psychiatry 4:43-48

30. Ryff CD, Keyes CL (1995) The structure of psychological wellbeing revisited. J Pers Soc Psychol 69:719-727

31. Beaton DE, Bombardier C, Guillemin F, Ferraz MB (2000) Guidelines for the process of cross-cultural adaptation of self-report measures. Spine 25:3186-3191

32. Smith BW, Zautra AJ (2004) The role of purpose in life in recovery from knee surgery. Int J Behav Med 11:197-202 
33. Vandecreek L, Paget S, Horton R, Robbins L, Oettinger M, Tai K (2004) Religious and non-religious coping methods among persons with rheumatoid arthritis. Arthritis Rheum 51:49-55

34. Bartlett SJ, Piedmont R, Bilderback A, Matsumoto AK, Bathon JM (2003) Spirituality, well-being, and quality of life in people with rheumatoid arthritis. Arthritis Rheum 49:778-783

35. Ryan RM, Deci EL (2001) On happiness and human potentials: a review of research on hedonic and eudaimonic well-being. Annu Rev Pyschol 52:141-166

36. WHOQOL SRPB Group (2006) A cross-cultural study of spirituality, religion, and personal beliefs as components of quality of life. Soc Sci Med 62:1486-1497
37. Scheier MF, Wrosch C, Baum A, Cohen S, Martire LM, Matthews KA et al (2006) The Life Engagement Test: Assessing purpose in life. J Behav Med 29:291-298

38. Van Dierendonck D (2005) The construct validity of Ryff's scales of psychological well-being and its extension with spiritual well-being. Pers Individ Differ 36:629-643

39. Astin JA, Beckner W, Soeken K, Hochberg MC, Berman B (2002) Psychological interventions of rheumatoid arthritis: a metaanalysis of randomized controlled trials. Arthritis Rheum 47:291-302

40. Riemsma RP, Kirwan JR, Taal E, Rasker JJ (2003) Patient education for adults with rheumatoid arthritis. Cochrane Database Syst Rev 2:CD003688 